\title{
Colour analysis and verification of CCTV images under different lighting conditions
}

\author{
R. A. Smith ${ }^{\mathrm{a}, \mathrm{b}}$, K. MacLennan-Brown ${ }^{* a, b}$, J. F. Tighe ${ }^{\mathrm{a}}$, N. Cohen ${ }^{\mathrm{a}}$, S. Triantaphillidou ${ }^{\mathrm{b}}$, \\ L. W. MacDonald ${ }^{\mathrm{c}}$. \\ ${ }^{a}$ Video Evidence Analysis Programme, Home Office Scientific Development Branch, \\ Sandridge, AL4 9HQ, UK \\ ${ }^{\mathrm{b}}$ Imaging Technology Research Group, University of Westminster, Harrow, HA1 3TP, UK \\ ${ }^{c}$ School of Printing \& Publishing, London College of Communication, \\ University of the Arts London, Elephant \& Castle, SE1 6SB, UK
}

\begin{abstract}
Colour information is not faithfully maintained by a CCTV imaging chain. Since colour can play an important role in identifying objects it is beneficial to be able to account accurately for changes to colour introduced by components in the chain. With this information it will be possible for law enforcement agencies and others to work back along the imaging chain to extract accurate colour information from CCTV recordings.

A typical CCTV system has an imaging chain that may consist of scene, camera, compression, recording media and display. The response of each of these stages to colour scene information was characterised by measuring its response to a known input. The main variables that affect colour within a scene are illumination and the colour, orientation and texture of objects. The effects of illumination on the appearance of colour of a variety of test targets were tested using laboratory-based lighting, street lighting, car headlights and artificial daylight. A range of typical cameras used in CCTV applications, common compression schemes and representative displays were also characterised.
\end{abstract}

Keywords: Colour reproduction, CCTV, video evidence, illumination

\section{INTRODUCTION}

On passing through an imaging chain, the colour information of an image is often not maintained accurately. ${ }^{1}$ This is particularly true for low-end imaging systems such as CCTV used for surveillance purposes. However, colour data can provide vital information, especially when working with low resolution imagery. A recent criminal case in the UK concerned video evidence in the form of a CCTV recording showing a car at night driving down a street lit by highpressure sodium lamps. Since poor image resolution meant that the vehicle registration plates were illegible, the issue of interest was whether the car captured in the video was the same colour as that of a suspect vehicle. ${ }^{2}$

This paper is the result of research into addressing such colour verification issues in general circumstances, with the aim of establishing a robust procedure to enable the accurate evaluation of colour information within CCTV recordings. A typical CCTV system has an imaging chain consisting of scene, camera, compression, recording media and display. Investigative work was carried out for each part of this chain, by characterising how accurately it reproduces a set of known surface colours.

The main variables within the scene are illumination, and the colour, orientation and texture of objects. The majority of this study was concerned with investigating the effect of common street illumination on the appearance of colour. Also tested were a number of different typical CCTV cameras of varying quality levels, different compression methods and two types of commonly employed display. Everything depends on the quality of the captured image, the deficiency of which is very difficult to recover later in the image processing chain. ${ }^{3}$

* Ken.maclennanbrown@homeoffice.gsi.gov.uk; phone +44 (0)1727816240; fax +44 (0)1727816253 
Several issues make it extremely difficult to accurately determine scene colour information from CCTV recordings. Firstly the scenes usually have uncontrolled lighting from a mixture of sources. Furthermore, the cameras are often of low quality and have not been characterised for their response to colour information. This problem is exacerbated by the huge number of different camera models in use designed by many different manufacturers. Once the images have been captured by the camera, they are stored either on VHS tape or, more commonly nowadays, on a hard disk in a proprietary digital video format. Analogue storage media and television encoding result in substantial loss of information.

This study is the first stage in establishing a robust method for assessing colour verification with video from CCTV systems. Once the effect of the imaging chain upon colour information is well understood, procedures can be developed to determine the colour properties of objects imaged in CCTV recordings.

\section{CCTV IMAGING CHAIN}

An imaging chain consists of the successive processes an image undergoes from capture to viewing. ${ }^{1}$ The variables considered most important in the particular imaging chain studied during this investigation are shown in Figure 1. Each aspect was investigated separately to understand its effect on overall colour fidelity.

- Scene - The two main components of a scene are the object in question and the lighting.

- Camera - The quality of camera can greatly affect the image fidelity. Sensor specification is the major factor but optical lens performance and on-board processing also play important roles.

- Compression - A lossy compression scheme can change colour information within an image.

- Recording Media - As the CCTV industry is increasingly dominated by digital technology, the impact of recording media has diminished. Since most data is now stored on hard disks, the media does not determine the format to such an extent as with analogue recordings, such as VHS tapes. Consequently, this part of the imaging chain was not investigated in depth.

- Display - The fidelity with which colour information is displayed to the end user depends upon the model of display being used and how it has been set up and calibrated.

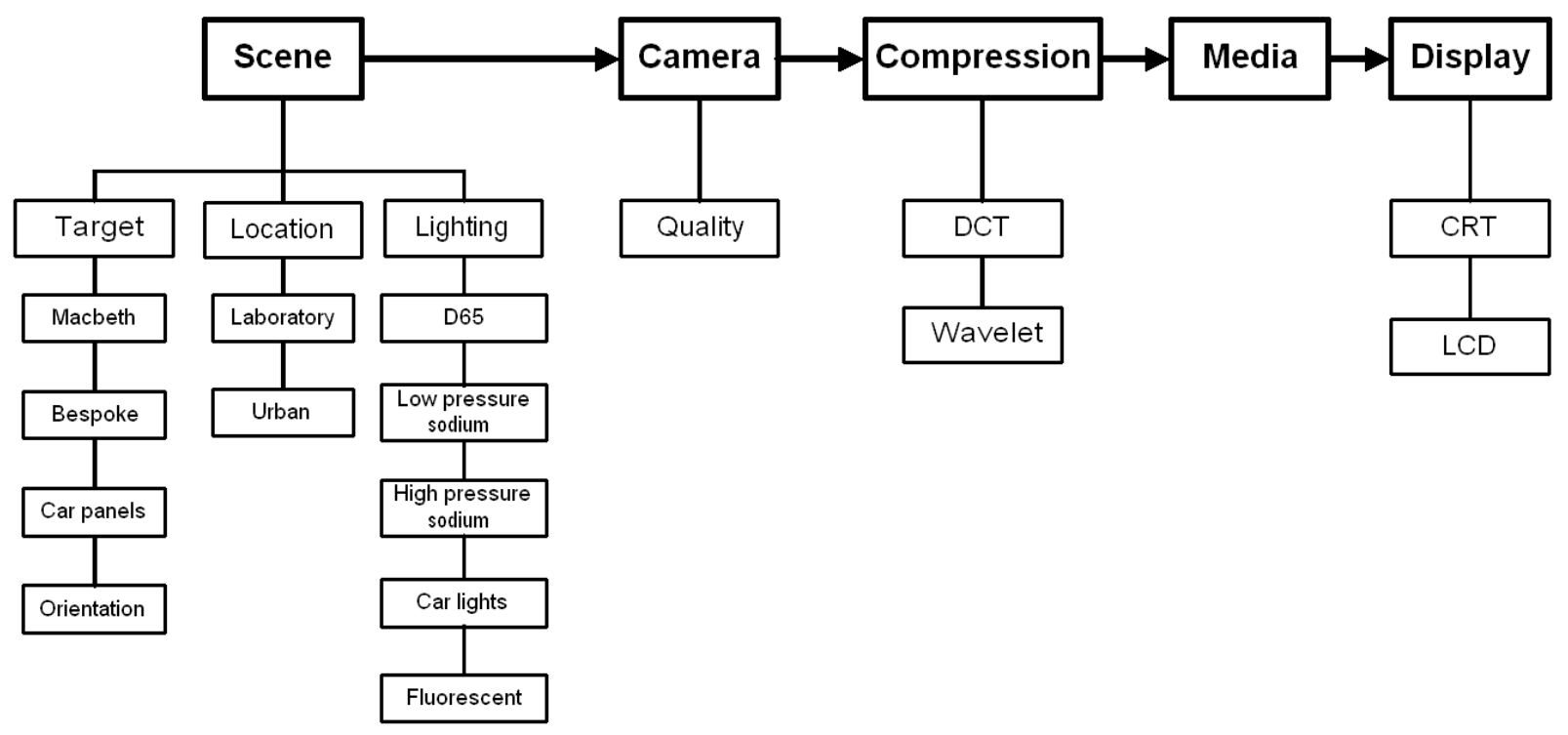

Figure 1 Aspects of the imaging chain studied in this investigation. 


\section{LIGHTING}

The effects on colour information of laboratory-based lighting, street lighting and daylight were examined. Since it is common in real-life scenes for a mixture of lighting to be present, the different forms of laboratory based lighting were also used in various combinations. The types of lighting used are listed in Table 1.

Table 1 Lighting sources used in investigation

\begin{tabular}{|l|l|l|}
\hline Laboratory-based & Street lighting & Car headlights \\
\hline D65 simulator & Low pressure sodium & Standard headlight bulb \\
\hline $\begin{array}{l}\text { Low pressure sodium Pure source } \\
\text { (LPS-Pure) }\end{array}$ & High pressure sodium & Super brilliance headlight bulb \\
\hline $\begin{array}{l}\text { Low pressure sodium Street source } \\
\text { (LPS-Street) }\end{array}$ & & Laser blue headlight bulb \\
\hline High pressure sodium (HPS) & & \\
\hline Fluorescent (F) & & \\
\hline
\end{tabular}

\subsection{Macbeth Color Checker Chart}

A spectroradiometer (Photo Research PR-650 SpectraScan) was used to measure the $X Y Z$ tristimulus value of each patch of a standard 24-patch Macbeth Color Checker Chart ${ }^{4}$ under each of the lighting conditions of Table 1. The chart was held at angles of both $90^{\circ}$ and $45^{\circ}$ to the spectroradiometer to take account of the fact that most real life objects are three dimensional and the angle at which light reflects off an object may change its apparent colour. Similar tests were performed using mixtures of the laboratory-based sources of Table 1 . Analysis of the measurements was done by converting the $X Y Z$ readings to CIE $x y Y, u^{\prime} v^{\prime}$ and $L^{*} a^{*} b^{*}$ colour spaces. ${ }^{5}$ For each of the lighting conditions the spectral response was calculated by using the spectral data from the white patch of the chart.

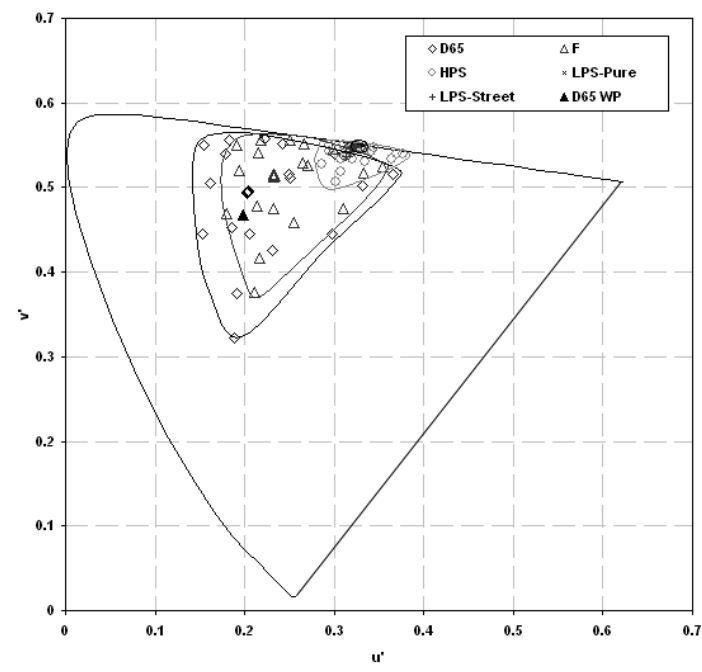

(a)

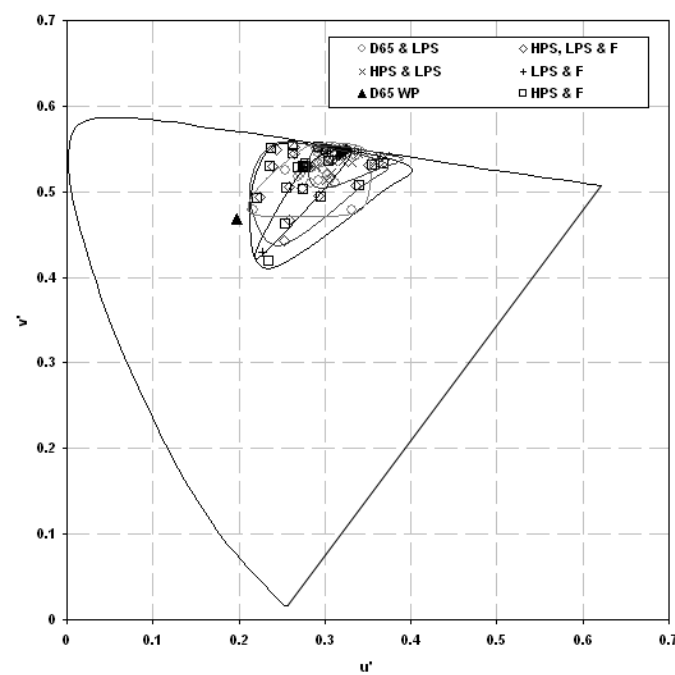

(b)

Figure 2 Chromaticity coordinates $\left(u^{\prime} v^{\prime}\right)$ for colour patches of the Macbeth Color Checker Chart (at $\left.90^{\circ}\right)$, measured under a variety of laboratory lighting conditions for: (a) single light sources; (b) combination of light sources. 
Figure 2a shows that the Macbeth Color Checker Chart (at 90 $)$ under D65 had the largest gamut of all lighting conditions tested, but this was small compared to the gamut of all possible colours (the boundary locus). When the chart was lit only by fluorescent lighting, the gamut was smaller and shifted slightly toward yellow. High pressure sodium lighting resulted in a much smaller gamut, while low pressure sodium lighting produced a gamut so small that all points virtually lie on top of each other and all useful colour information is lost. This is an indication of the very poor colour rendering properties of low pressure sodium light, producing an almost monochrome image.

It is worth noting in Figure $2 b$ that when different lighting sources were mixed, colour chromaticities were affected with the resultant gamut from the mixed lighting falling between the gamuts of the contributing sources, according to their relative intensities. Although an in-depth study of varying the respective contributions was not undertaken, we can reasonably assume that the behaviour of composite light sources could be modelled by additive combination.

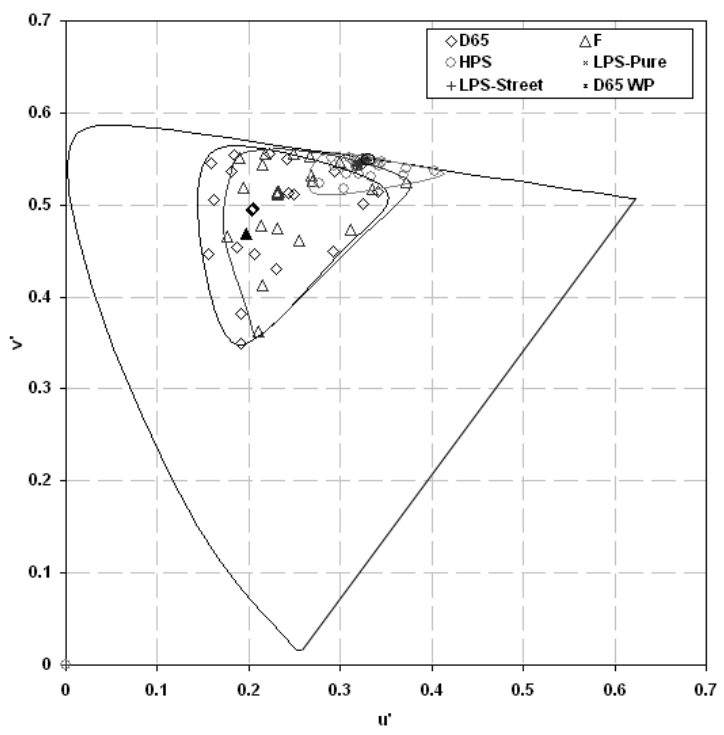

(a)

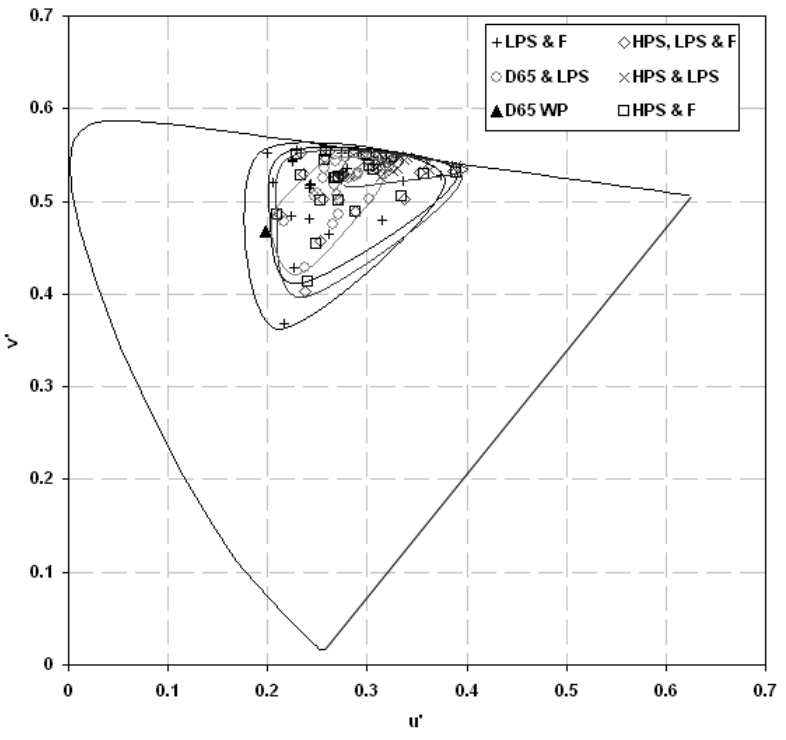

(b)

Figure 3 Chromaticity coordinates ( $\left.u^{\prime} v^{\prime}\right)$ for colour patches of the Macbeth Color Checker Chart (at $\left.45^{\circ}\right)$, measured under a variety of laboratory lighting conditions for: (a) single light sources; (b) combination of light sources.

When the Macbeth Color Checker Chart was held at $45^{\circ}$ to the spectroradiometer, results similar to those of Figure 2 were obtained, although there were slight differences in absolute values as shown in Figure 3 . This indicates that orientation of objects may play an important role in their apparent colour.

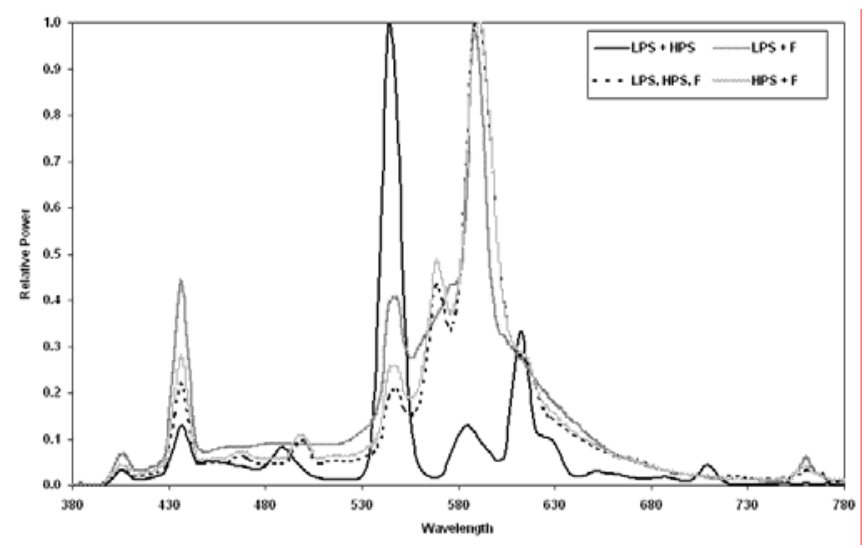

Figure 4 Spectral responses of the white patch of Macbeth Color Checker Chart under combinations of light sources 
The normalised spectral responses measured from different combinations of lighting sources are shown in Figure 4. Both the HPS \& F and HPS, LPS \& F responses follow similar curves with clear peaks, with the one containing LPS being displaced slightly below the other. The LPS \& F curve contains similar features to the HPS \& F and HPS, LPS \& F curves. The LPS \& HPS curve is the only one to differ significantly, indicating that the fluorescent lighting dominated the other sources in the combination studied.

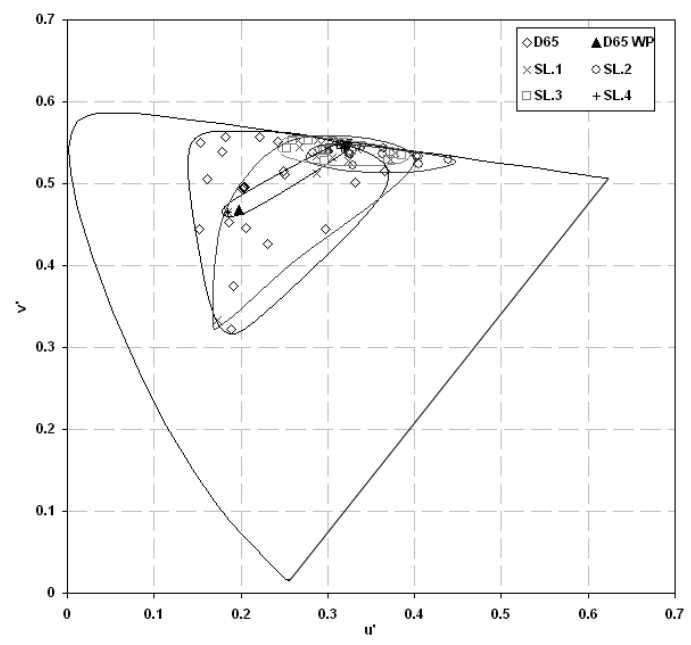

Figure 5 Chromaticity coordinates from Macbeth Chart $\left(\right.$ at $\left.90^{\circ}\right)$ under four different street lighting conditions (SL1 - 4).

The effect of using real-life lighting conditions is shown in Figure 5, highlighting that when street lighting was the primary source of light, only a small gamut of colours was obtained. From the gamut and chromaticity coordinates alone it would not be possible to state with certainly what type of lighting has been used.

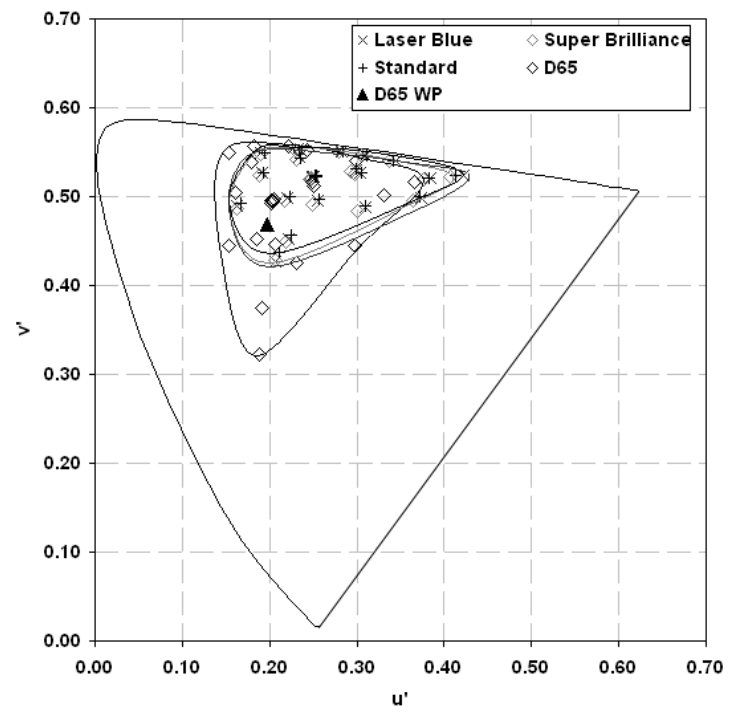

Figure 6 Colour information from Macbeth Colour Checker Chart (at $\left.90^{\circ}\right)$ under different car headlights

Slightly more colour information was retained when car headlights were used. Figure 6 shows gamuts of the Macbeth Color Checker Chart under three types of car headlights. All three of the headlights produced similar gamuts. 


\subsection{Car Panels}

Twenty-four A6 sized sheets of $2 \mathrm{~mm}$ steel were painted in a variety of colours to represent a pool of real-life car examples to be used as test targets. Even within a specified car colour there are further variables to consider such as:

- Paint manufacturer

- Age of the paint

- Composition of the paint

- After care treatment to paint

It was decided to standardise on one paint manufacturer (Halfords), to use a selection of both block and metallic paints, and to treat all painted surfaces with wax. The colours of car panels are shown in Table 2. The XYZ values of these panels were measured using a spectroradiometer under the laboratory-based lighting conditions of Table 1 .

Table 2 Colours of test car panels

\begin{tabular}{|l|l|l|l|}
\hline BMW Jet Black & Rover Midnight Blue & Ford Purple Velvet & Peugeot Cherry \\
\hline BMW Cosmos Black & Ford Royal Blue & Peugeot Conifer & Brilliant Orange \\
\hline Volvo Dark Grey & Fiat Capri Blue & Rover Brooklands Green & Fiat Broom Yellow \\
\hline Ford Graphite Grey & Ford Olympic Blue & Rover Applejack & Ford Jasmine \\
\hline Rover Gunmetal & Ford Metropolis Blue & Rover Maple & Vauxhall Gazelle Beige \\
\hline Ford Moondust Silver & Ford Bermuda & Volkswagen Gambia Red & Mercedes Polar White \\
\hline
\end{tabular}

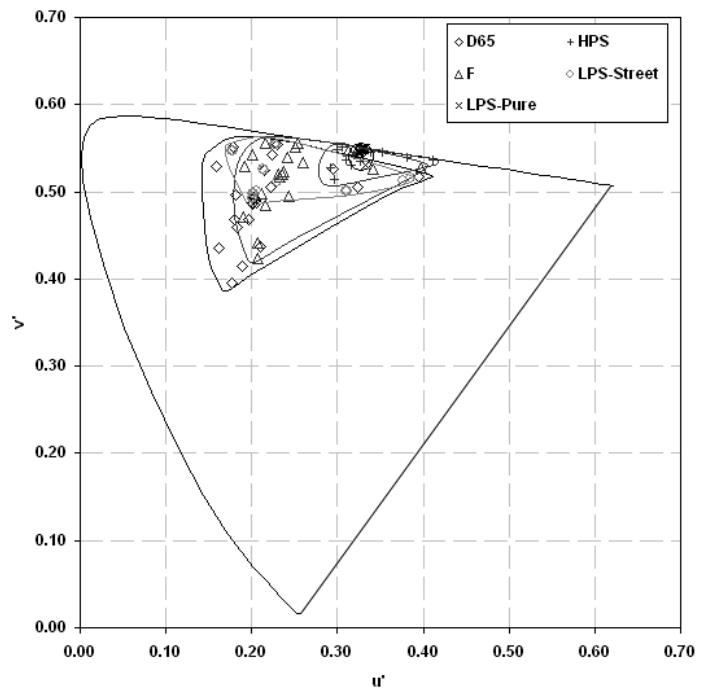

(a)

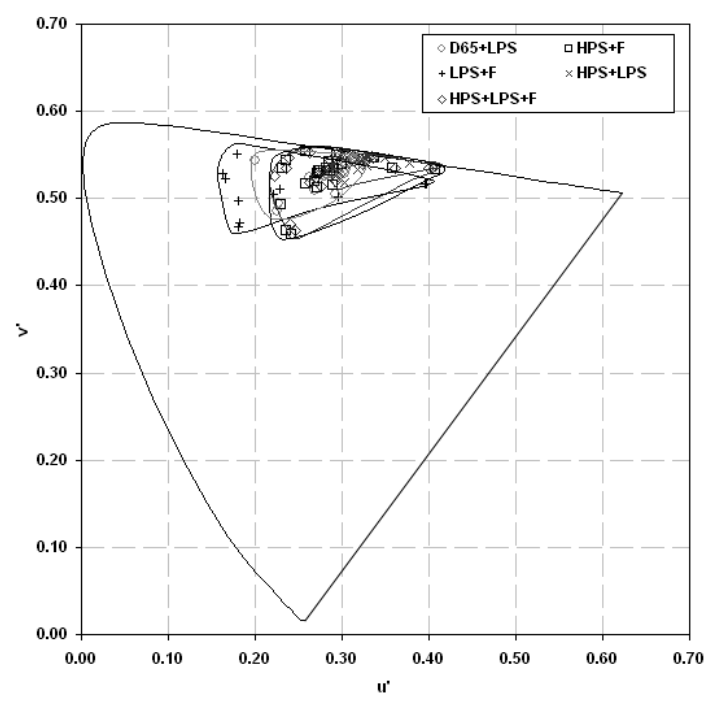

(b)

Figure 7 Chromaticity coordinates $\left(u^{\prime} v^{\prime}\right)$ for painted metal panels $\left(\right.$ at $\left.90^{\circ}\right)$, measured under a variety of laboratory lighting conditions for: (a) single light sources; (b) combination of light sources.

Figure 7 shows the $u^{\prime} v^{\prime}$ values converted from the spectroradiometer readings. Whilst the results were broadly similar to those found using the Macbeth Colour Checker Chart (Figure 2), it is interesting to note that the gamut obtained with the car panels under LPS-Street lighting was larger than that of the Macbeth Colour Checker Chart under similar lighting conditions. Of course this is still a drastically reduced gamut when compared with the panels viewed in daylight. 


\section{CAMERA}

As it was possible to test only a very small sample of the many available CCTV cameras on the market, it was decided to use representative examples from across the price range. In descending order of price, these were a Sony ExWave, a Baxall Ice, a Vista Protos and an X-Vision. The PAL-encoded video signal output by all cameras was analysed.

For each camera, the Macbeth Color Checker Chart was placed so that it filled the entire field of view. The exposure and focus were set to the optimal values for each camera and lighting combination. A one-minute video sequence was then recorded onto DVCPro tape under the laboratory lighting combinations listed in Table 1. A ten-second clip approximately in the middle of each recording was selected and three bitmap images were taken from this clip. On comparing these, the only visible variation was the superimposed noise. The still images were then displayed on an LCD monitor and the $X Y Z$ value of each patch was measured with the spectroradiometer.

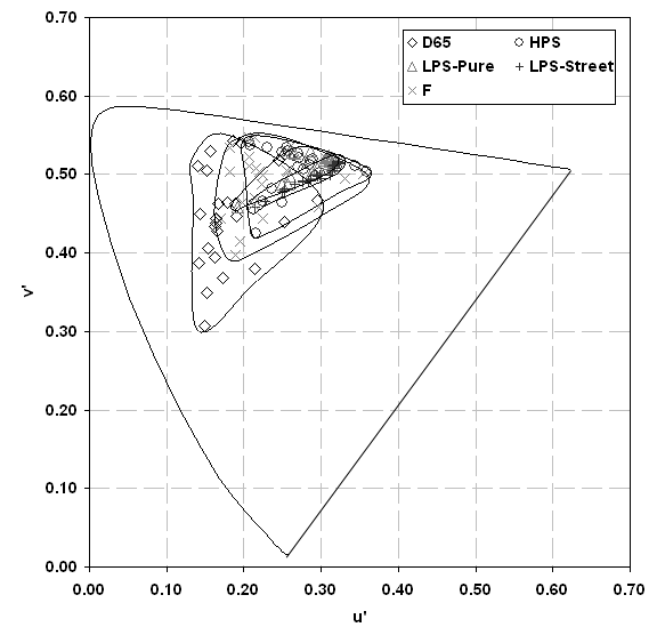

(a)

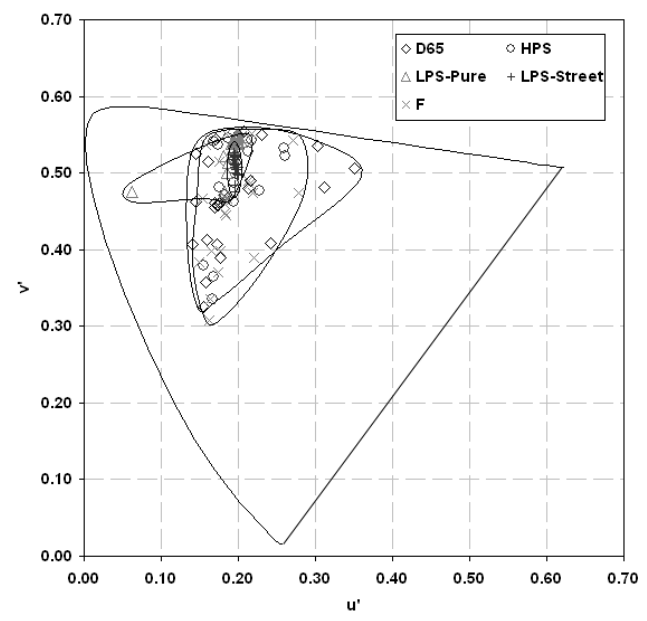

(c)

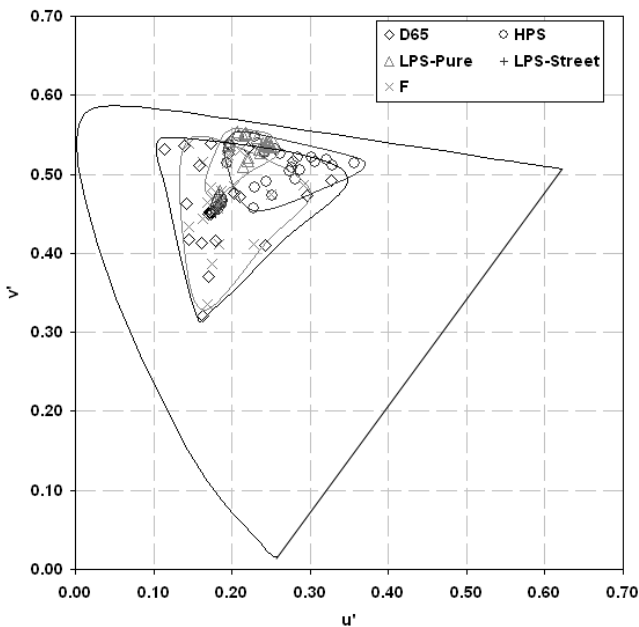

(b)

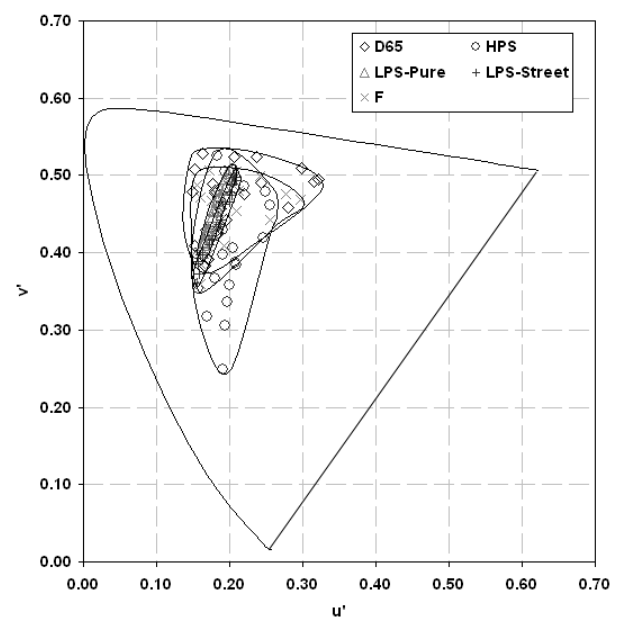

(d)

Figure 8 Chromaticity coordinates from Macbeth Color Checker Chart captured under single light sources via four CCTV cameras: (a) Sony ExWave; (b) Baxall Ice; (c) Vista Protos; (d) X-Vision. 
Figure 8 gives a clear indication of how the quality of the camera impacted on the colour information obtained. This was particularly noticeable with the results from the cheapest camera, manufactured by X-Vision, shown in Figure 8d where the gamuts can be seen to be considerably reduced.

\section{COMPRESSION}

To test the effect of compression in the imaging chain, a bespoke test target was generated comprising red, green, blue, cyan, magenta, yellow and luminance step wedges made up of 11 patches starting at a value of 5 in the relevant channel (one or more of RGB) and increasing with a step value of 25 to a maximum value of 255, as shown in Figure 9 . While the effects of compression were obviously scene dependent ${ }^{6,7}$, this simple, static, test target was chosen as it was only the effect on colour that was of interest.

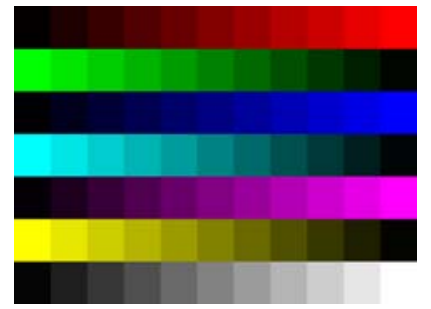

Figure 9 Bespoke test target used for testing colour fidelity in compression

Generic compressors for DCT and Wavelet schemes were investigated. For the DCT scheme, the JPEG compression function of MATLAB was used, as this is similar to the compression commonly found in digital CCTV systems. A JPEG2000 Photoshop plug-in was used for the Wavelet scheme. Each compressor was run at five quality levels of 'Maximum', 'High', 'Medium', 'Low' and 'Minimum' on the bespoke test target of Figure 9. The corresponding compression ratios are shown in Table 3. The original bitmap image was then compared with the compressed images and differences analysed. To take account of artefacts, this comparison was performed after averaging RGB values over each patch area of the compressed image.

Table 3 Quality level and corresponding compression ratios used in investigation

\begin{tabular}{|c|c|c|}
\hline Quality Level & $\begin{array}{c}\text { Compression ratio for } \\
\text { JPEG }\end{array}$ & $\begin{array}{c}\text { Compression ratio for } \\
\text { JPEG2000 }\end{array}$ \\
\hline Max & $1: 30$ & $1: 68$ \\
\hline High & $1: 56$ & $1: 68$ \\
\hline Med & $1: 68$ & $1: 119$ \\
\hline Low & $1: 87$ & $1: 158$ \\
\hline Min & $1: 135$ & $1: 237$ \\
\hline
\end{tabular}




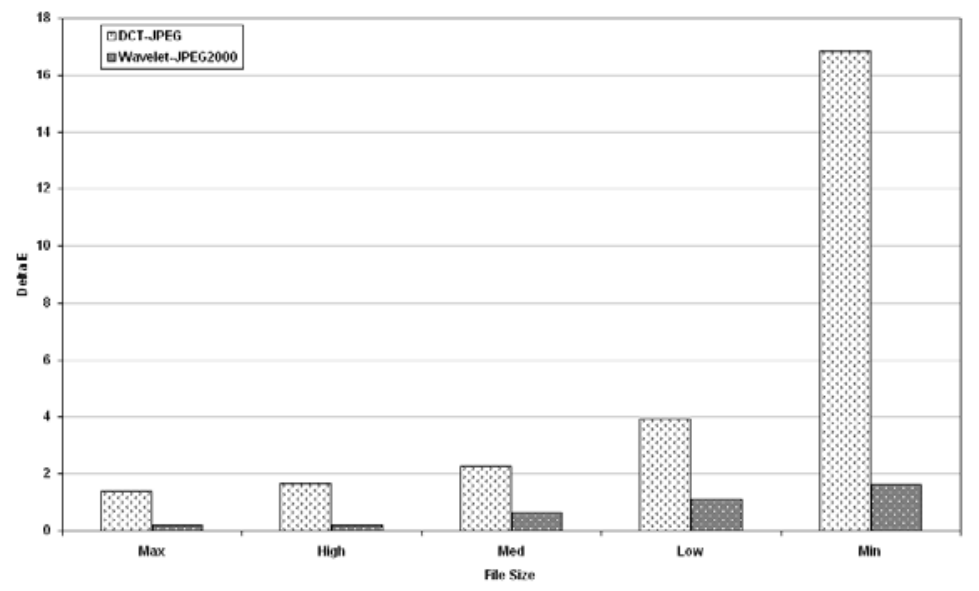

Figure 10 Average $\Delta E^{*}$ between original and DCT and Wavelet compressed images for different degrees of compression

Figure 10 shows the average colour error, expressed as Delta $\mathrm{E}\left(\Delta E^{*}{ }_{a b}\right)$ between the original and compressed images for both DCT (JPEG) and Wavelet (JPEG2000) compression at different quality settings. For both compression methods it is obvious that as the quality and file size decreased, the average value of Delta E increased due to the larger number of artefacts, although the Wavelet value was always less than its DCT equivalent. There was a particularly dramatic jump in average Delta E value between the Low and Minimum settings for the DCT compression, which was not matched by the Wavelet compression. This illustrates that DCT seems to be a more aggressive compressor than Wavelet, resulting in greater loss of image quality, whilst the latter produces smaller file sizes.

\section{DISPLAY}

Two Sony computer displays, one CRT and one LCD, were tested to represent typical models in common usage. For the characterisation of these displays, a series of test targets was created varying in colour and intensity. Each test target consisted of five patches, one placed in each of the four corners and one in the centre of the screen. The total area of these patches was half of the total area of the image to ensure that the power requirements of the display were similar for all images. In total there were four sets of test targets (red, green, blue and achromatic), each containing 52 images. Each set started with a black and white target, with each subsequent image in the set increasing in RGB intensity by 5 units of the relevant colour for the patches, with the background of the target displaying the corresponding inverse RGB value. Examples from one set are shown in Figure 11.

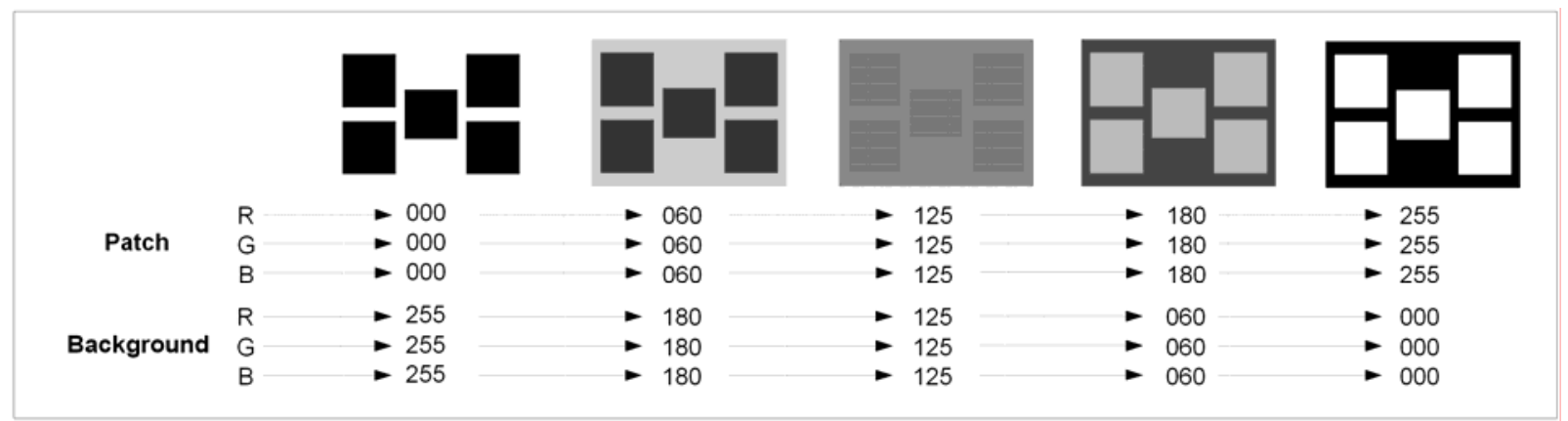

Figure 11 Examples from achromatic set of test targets with RGB values of patches and background.

Before any measurements were taken, the computer displays were calibrated with a colour management device (Pantone ColorVision SpyderPRO) according to the manufacturer's instructions. For the Sony CRT display, ${ }^{8,9}$ the test targets were displayed and a spectrophotometer (Gretag Macbeth Spectrolino) was used to take five measurements (which where then averaged) from the centre of each of the five patches of each image. Since the spectrophotometer could not read the Sony LCD monitor at low intensity values, the spectroradiometer was used to take corresponding measurements for this display. ${ }^{10}$ 


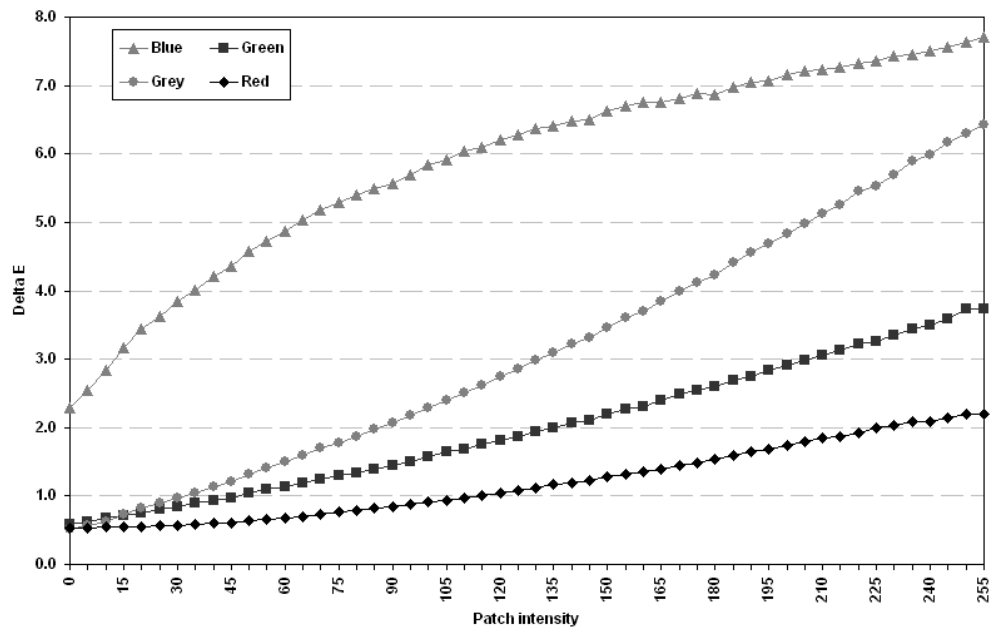

Figure 12 Graph showing average colour variation (Delta E) across screen for the CRT display.

The data was analysed by first converting the output $X Y Z$ measurements to $L * a * b *$ colour space, and then calculating the Delta $E$ between the various patches. These values were then averaged to quantify the variation across the display. The Delta E results for the CRT monitor are shown in Figure 12, indicating that the variation was dependent on both signal level and colour channel. The intensity-lightness response curves for the CRT and LCD monitors are shown in Figure 13. It is apparent that the LCD monitor has a more linear response, which may lead to an improved ability to reproduce colour.

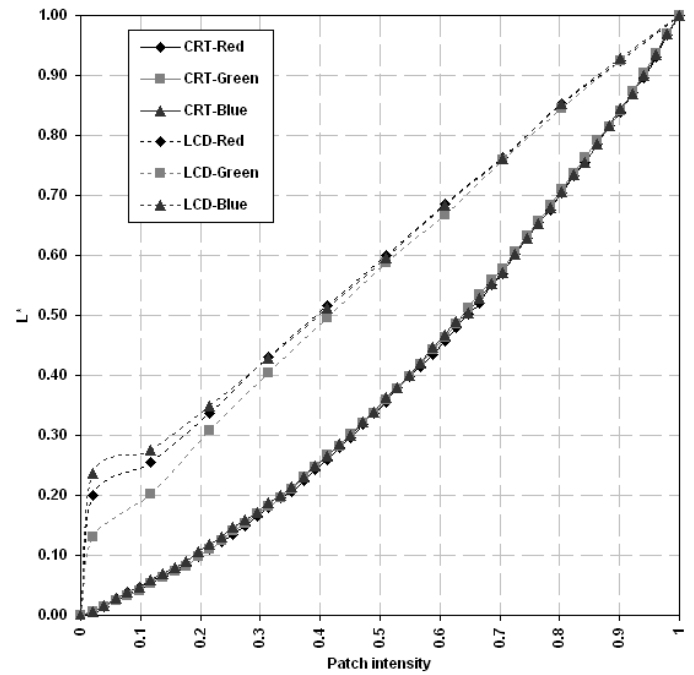

Figure 13 Intensity-lightness response curves for Sony CRT and LCD computer displays.

\section{CONCLUSIONS}

The impact of each part of the imaging chain on colour reproduction was analysed by converting the measurements into CIELAB colour space and by plotting the gamut for each lighting condition. It is apparent that the type and level of illumination can have the greatest effect on the colour information that is obtained from the image. The results show that when a scene is lit with sodium lighting, the gamut of possible colours is considerably reduced. The investigation on compression confirms that the reproduction of colour is dependent on the scheme employed and the degree of 
compression. It can be concluded from the investigation into cameras, that there is a strong correlation between camera performance and colour fidelity. This study indicates that in many situations it will be possible to extract useful colour information from CCTV recordings once appropriate corrections have been made. However, in circumstances where the scene has been lit with sodium lighting, of both high-pressure and low-pressure types, there is not enough colour information present in the scene to enable later extraction of meaningful colour data.

\section{REFERENCES}

R.W.G. Hunt, The Reproduction of Colour, $5^{\text {th }}$ Edition, 1995, Fountain Press, England.

2 L.W. MacDonald, "Fatal Flaws: Uncertainty in the Interpretation of Colour in CCTV Images", Annals of the British Machine Vision Association (BMVA) 2007, (7) 1-11. http://www.bmva.ac.uk/annals/2007/2007-0007.pdf

3 M. Bromby and H. Ness “Over-observed? What is the Quality of this New Digital Legal World?", Proc. 20th BILETA Conf., Belfast, 2005.

4 C.S. McCamy, H. Marcus and J.G. Davidson, "A Color Rendition Chart," Journal of Applied Photographic Engineering 11(3) Summer issue, 1976, 95-99.

5 R.W.G. Hunt, Measuring Colour, $3^{\text {rd }}$ Edition, 1998, Fountain Press, England.

6 E. Allen, S. Triantaphillidou and R.E. Jacobson, "Image quality comparison between JPEG and JPEG2000. I. Psychophysical Investigation", Journal of Imaging Science and Technology, 51, 2007.

$7 \quad$ S. Triantaphillidou, E. Allen and R.E. Jacobson, "Image quality comparison between JPEG and JPEG2000. II. Scene dependency, scene analysis and classification", Journal of Imaging Science and Technology, 51, 2007.

8 British Standard: 61966-3:2000, Multimedia systems and equipment - Colour measurement and management - Part 3: Equipment using cathode ray tubes.

9 A.M. Ford et al, Assessment of a CRT Display System, Journal of Photographic Science, Vol. 44, 1996.

10 British Standard: 61966-4:2000, Multimedia systems and equipment - Colour measurement and management - Part 4: Equipment using liquid crystal display panels. 